\begin{tabular}{|c|c|}
\hline \multirow{3}{*}{ 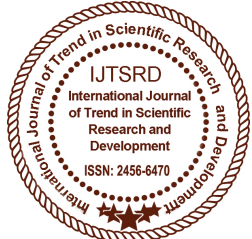 } & International Journal of Trend in Scientific Research and Development (IJTSRD) \\
\hline & International Open Access Journal | www.ijtsrd.com \\
\hline & ISSN No: 2456 - 6470 | Volume - 2 | Issue - 6 | Se \\
\hline
\end{tabular}

\title{
Approach Towards Analysis of Biopsy Slide of Breast Cancer
}

\author{
Dr. Indra Kanta Maitra ${ }^{1}$, Prof. Samir Kand Bandyopadhyay ${ }^{2}$ \\ ${ }^{1}$ Assistant Professor, B. P. Poddar Institute of Management and Technology, Kolkata, West Bengal, India \\ ${ }^{2}$ Professor, Advisor to Chancellor, JIS University, Kolkata, West Bengal, India
}

\begin{abstract}
Pathologists placed tissue slices on glass slides use appropriate stains and examine them through a microscope. The proposed method uses insignificant portions of the slide images by colour polarization as a pre-processing step. The simplicity of algorithm leads to less computational time and thus suitable tool to assist experts for automated real-time breast cancer diagnosis.
\end{abstract}

Keyword: Histopathological slides, Hyperplasia, Situ, Invasive Cancer

\section{INTRODUCTION}

Image processing techniques play an important role towards the interpretation of histopathological slides like breast biopsies. Several researches have been started worldwide for automatic interpretation of histopathological slide. Most of them are very much successful to assist the expert to interpret the abnormalities. Due to the growth in computational speed and advanced image processing algorithm digital pathology is now a reality specially for identification of abnormalities.

Cancer can develop from normal cells that go through abnormal mutation that eventually transformation those to malignant cells which reproduce out of control. Many breast cancers grow from a sequence that initiates with an increase in the number of breast cells (hyperplasia) to the beginning of unusual breast cells (atypical hyperplasia) followed by carcinoma in situ (non invasive cancer) and finally, invasive cancer. Not all breast cancers necessarily follow the same pattern. The speed of advancement for those is highly inconstant. It is also evident that some cancers may never progress beyond in situ disease [1]. Before further progress to describe the proposed method, it is important to understand the types and characteristic features of the different categories of breast cancer that can be interpreted by the biopsies.

Lobular Carcinoma in Situ (LCIS) - Lobular cancer in situ (LCIS) means that there are cells change inside the breast lobes. This is not cancer. A patient having LCIS means that patients have an increased risk of getting breast cancer in the future.

Ductal Carcinoma in Situ (DCIS) - Ductal cancer in situ (DCIS) means that cells inside some of the ducts of breast have started to turn into cancer cells.

Invasive Ductal Breast Cancer (IDBC) - Invasive Ductal Breast Cancer is the most common type of breast cancer. Between 70 and 80 out of every 100 breast cancers diagnosed are of this type.

Invasive Lobular Breast Cancer (ILBC) - About 1 in 10 breast cancers diagnosed are of invasive lobular carcinoma.

Beside the aforesaid four types of breast cancer there are several other categories of invasive breast cancer that have been reported namely Tubular carcinoma, Medullary carcinoma, Mucinous carcinoma, Meta plastic carcinoma, Invasive cribriform carcinoma, Invasive papillary carcinoma and Invasive micro papillary carcinoma. These categories have their own identifiable characteristic features and originated in specific part of the breast. Instead of invasive breast cancer, there are also some other types of cancers and are also developed in breast like Inflammatory breast cancer, Paget's disease of the nipple, Phylloidestumors etc.

The small percentage of breast cancers that are not identified by mammography may be missed out as mammography uses $\mathrm{x}$-ray machines designed 
specially to image the breasts. Breast MRI also requires special equipment for breast imaging. Higher-quality images are produced by dedicated breast MRI equipment than by general purpose machines designed for head, chest or abdominal MRI scanning. It is important that screening MRIs are done at facilities that are capable of performing an MRIguided breast biopsy at the time of the examination if abnormalities are found.

Sometime interpretations of mammograms can be difficult because a normal breast of different women may vary significantly. Also, the appearance of an image may be compromised if there is powder or salve on the breasts or if they have undergone breast surgery. Some breast cancers are difficult to visualize. Not all cancers of the breast can be seen on mammography [2].

The proposed algorithm leads to less computational time and thus suitable tool to assist experts for automated real-time breast cancer diagnosis.

\section{Literature Review}

The histopathological slide image analysis of digital microscope is a young subject with enormous potentiality. There are several interesting works that have been carried out by different research groups. But most of the research is concentrated in the field of blood sample analysis and decision support system. In the field of tissue culture or more specifically cancer detection by biopsy slide is highly dependent on human intervention.

Automatic CAD system development in this field has not initiated much. There are some researches but they are mostly theoretical in nature. In this paper, few of the researches are discussed below which are dealing with this related field.

Some Researchers reported a computational method that modelled a type of brain cancer using topological properties of cells in the tissue image. They constructed the graphs based on the locations of cells within the image. They used the Waxman model in their experiment [3-5].

Researchers also classified [6-7] the breast cancer tissues using graph theory. These approaches towards automatic detection of cancer actually failed because the types of cancers identified were more complicated.
Other researchers introduced a Grid-enabled CAD to perform automatic analysis of imaged histopathology breast tissue specimens [8]. More than 100,000 digitized samples $(1200 \times 1200$ pixels $)$ were processed on the Grid. They analyzed results for 3744 breast tissue samples, which were originated from four different institutions using diaminobenzidine (DAB) and hematoxylin staining.

Another researcher presented a computationally efficient method for analyzing $\mathrm{H} \& \mathrm{E}$ stained digital pathology slides with the objective of discriminating diagnostically relevant vs. irrelevant regions [9].

Other Researcher proposed a computer aided diagnosis system dedicated to virtual microscopy based on stereo logy sampling and diffusion maps. The original strategy is presented, combining stereological sampling methods based on test grids and data reduction methods based on diffusion maps, in order to build a knowledge image database. The practical application of the exposed methodology concerns virtual slides of breast tumors [10].

\section{Proposed Method}

The proposed method is an introductory preprocessing work towards the confirmation of breast cancer using histopathological slide of biopsy. Digital slide image analysis is a complex work and required long time to establish viable algorithm to detect the abnormalities in a slide without further human intervention. The proposed work is preliminary initiative towards the same. It identifies the abnormalities but as a pre-processing step it enhances the histopathological slide, so that, if abnormalities present, it will become more noticeable. It will make easy the search for abnormalities by the experts of this field.

In the proposed work, free Tissue Blocks downloaded from OriGene Technologies [2] are used as dataset. In the experiments, breast cancer tissues from different patients and non- cancerous breast tissues from different normal females are considered. The sample images are 24-bit bitmap image with the size of 640X480 Pixels.

The sample images provided by the OriGene technologies are coloured image. The coloured image is more informative but at the same time it will increase the complexity of the method towards analysis of the image. The 24-bit bitmap can produce 
almost 16,777,216 number distinct colours shades which are virtually impossible to handle. So, conventionally almost all the medical image processing algorithms use the grey shade image to reduce the complexity of the method and preserve maximum information within the image to investigate.

The grey image contains 256 numbers of grey shades to represent an image. The proposed method analyzed different alternative methods to convert the colour image to grey scale image. The most important factor here is to preserve the relative colour distance within

$$
P=\sum_{t=1}^{h} \sum_{j=1}^{w} I_{t, j}
$$

Now the Euclidean distances (ED) are calculated for all the colour shade in the GP.

$$
E D_{i}=\sqrt{\sum_{i=1}^{n}\left(G P_{i}-P \cdot R\right)^{2}+\left(G P_{i}-P \cdot G\right)^{2}+\left(G P_{i}-P \cdot B\right)^{2}} \text { Where } n=255
$$

The grey shade with lowest distance $\left(\mathrm{ED}_{\mathrm{i}}\right)$ is selected and value of the grey shade is propagated to red, green and blue component of the pixel, ensuing the conversion from colour to grey scale image. The iteration will terminate when all the pixels of the colour image are converted to grey scale.

\section{Algorithm: Grey Scale Conversion}

\section{GREY-SCALE-CONVERSION (I, Height, Width)}

I $\leftarrow$ Sample Image

$P \leftarrow$ Single Pixel

Loop $i \leftarrow 1$ to Height

Do Loop $j \leftarrow 1$ to Width

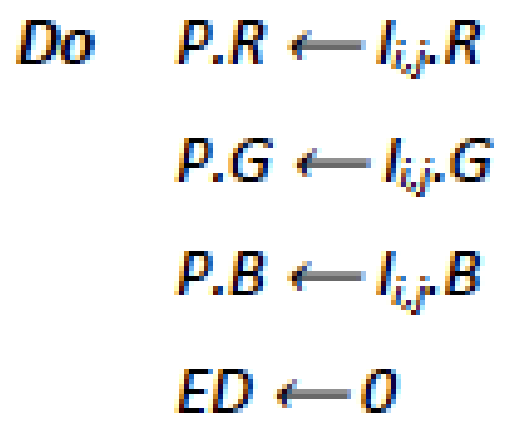

$\operatorname{MaxV} \leftarrow 0$

$\operatorname{MinV} \leftarrow 255$ 
// Comparing Euclidean distance

Loop $k 0$ to 255

Do If $E D \leq S Q R T\left((k-P . R)^{\wedge} 2+(k-P . G)^{\wedge} 2+(k-P . B)^{\wedge} 2\right)$

Then $R \leftarrow k$

$I_{i, j} R \leftarrow R$

$I_{i, j} \cdot G \leftarrow R$

$I_{i j} . B \leftarrow R$

// Calculating the minimum and maximum intensity value

If $k \leq \operatorname{MinV}$

Then MinV $\leftarrow R$

If $k \geq \operatorname{MaxV}$

Then MaxV $\leftarrow R$

\section{Return I}

The proposed method is implemented using three loops. The outer loop is for height, inner loop is for width and the inner most loop is to traverse the grey scale palette. Assuming that height $=$ width $=\mathrm{n}$ and grey palette is $\mathrm{k}$. So, the running time of the algorithm is $\mathrm{k} . \mathrm{n}^{2}$. But the grey palette i.e. $\mathrm{k}$ is always 256 , so, it is a constant. Hence, the running time of the algorithm is order of $n^{2}$.

The initial objective of the process is to eliminate the irrelevant object from the slide to make it more enhanced and clear. The final objective is to merge the similar objects in slide by colour polarisation technique. The initial objective will be achieved by increasing the contrast level of the slide by using a constant $(\Delta)$ with the intensity shades. The final objective will be accomplished by colour polarisation using a threshold (k) determined by the central tendency of image. For simplicity, the average of highest (MaxV) and lowest (MinV) intensity of colour can be used for thresholding which are already derived in previous algorithm.

$$
k=(\operatorname{Max} V+\operatorname{MinV}) / 2
$$

Finally, the inverse image is generated due to better understanding of the abnormalitiespresent in the sample image.

$$
P_{i, j}=255-P_{i, j}
$$

\section{Algorithm: Colour Polarisation}

COLOUR-POLARISATION (I, Height, Width, MaxV, Mir

$I \leftarrow$ Sample Image

$P \leftarrow$ Single Pixel 


$$
k \leftarrow(\operatorname{Max} V+\operatorname{MinV}) / 2
$$

Loop $i \leftarrow 1$ to Height

\title{
Do $\quad$ Loop $j \leftarrow 1$ to Width
}

\author{
Do $\quad V a l \leftarrow I_{i j}$ \\ // Enhancing the contrast \\ If $\mathrm{Val} \geq 128$

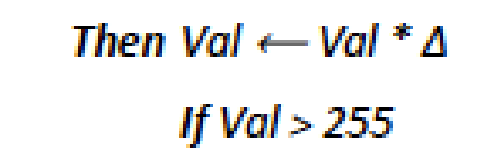

Then $\mathrm{Val} \leftarrow 255$

Else $\mathrm{Val} \leftarrow \mathrm{Val} / \Delta$

If $\mathrm{Val}<0$

Then $\mathrm{Val} \leftarrow 0$

// Merging the similar pixels by colour polarisation

If $\mathrm{Val} \geq k$

$$
\begin{aligned}
& \text { Then Val } \leftarrow 255-\text { MaxV } \\
& \text { Else Val } \leftarrow 255-\text { MinV } \\
& I_{i j} \leftarrow \text { Val }
\end{aligned}
$$

\section{Return I}

The proposed method traverses the entire image. The outer loop is used for the height whereas the inner loop is for width. Assuming that, height $=$ width $=n$. Then the running time of the algorithm is order of $n^{2}$.

\section{Experimental Result}

The primary objective of the proposed method is to remove the huge amount of fat, connective tissue and gland tissue from the cancerous cells within the histopathological biopsy samples. The stage, intensity, type, future development and treatment of cancer can only be detected on the basis of orientation of malignant cell, shape of the cell and duct, density carcinogenic cells in compare with normal cells. The outputs of aforesaid algorithms are depicted in the figure 1 to figure 6 for cancerous cells within the biopsy slide and normal slide along with the histogram of the images.
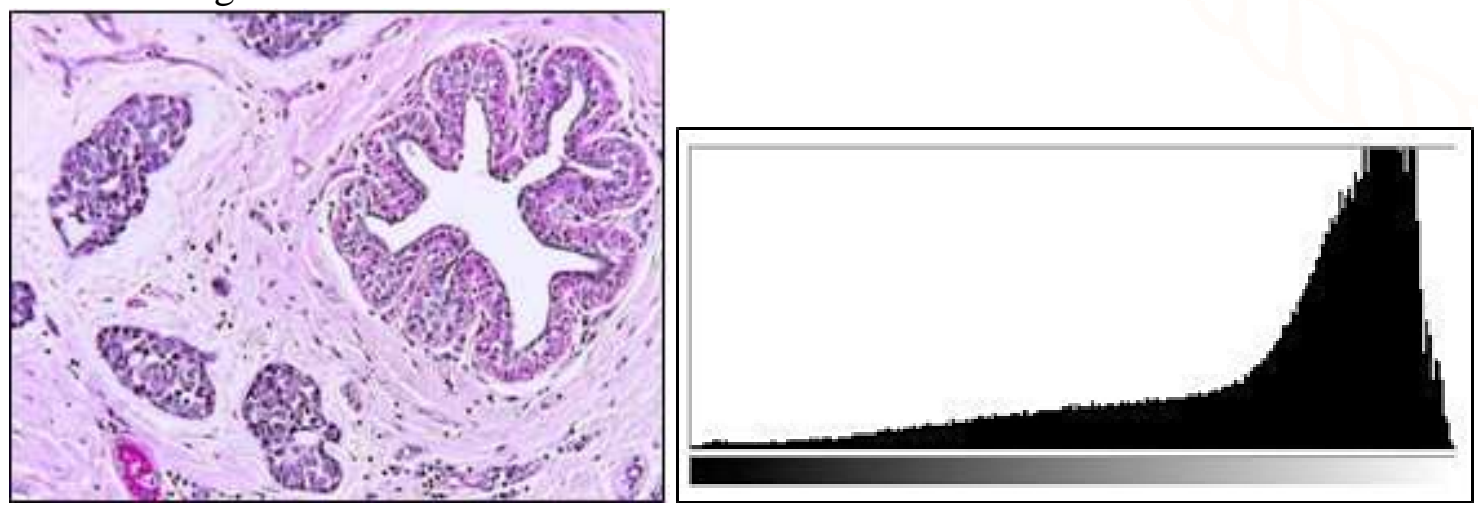

Figure 1 The Original Histopathological Slide showing Malignant Cells along with Histogram 
International Journal of Trend in Scientific Research and Development (IJTSRD) ISSN: 2456-6470
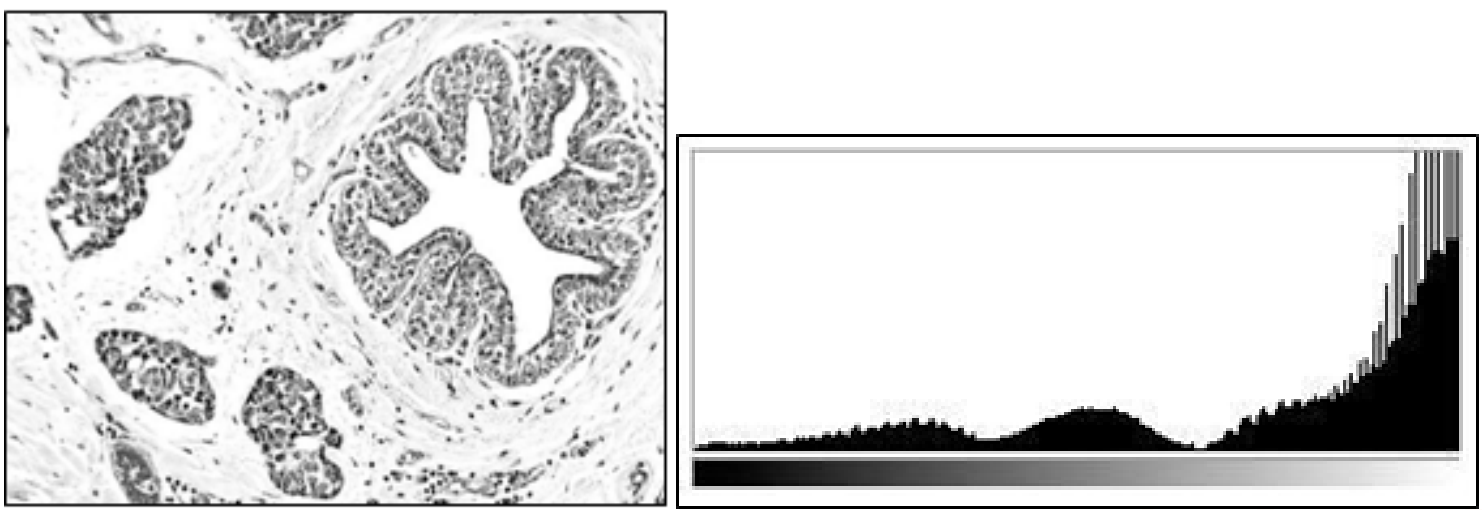

Figure 2 The Grey Shade Histopathological Slide showing Malignant Cells along with Histogram

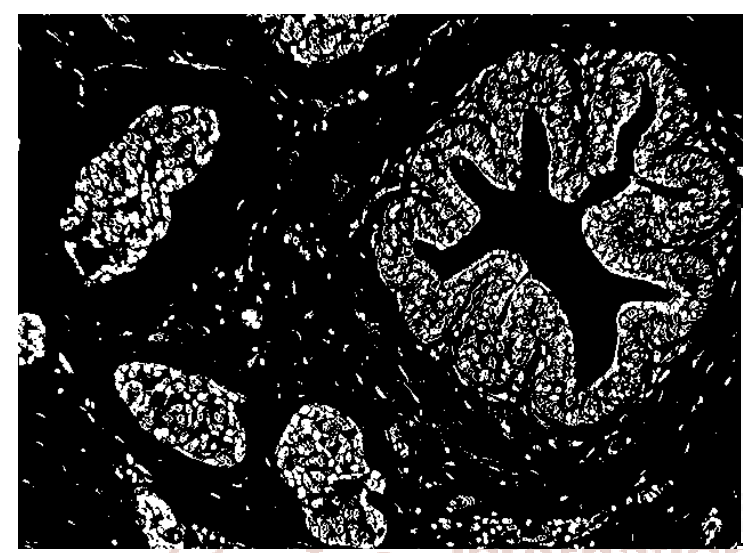

Figure 3 Inverse ColourPolarisedHistopathological Slide showing EnhancedMalignant Portion along with Histogram
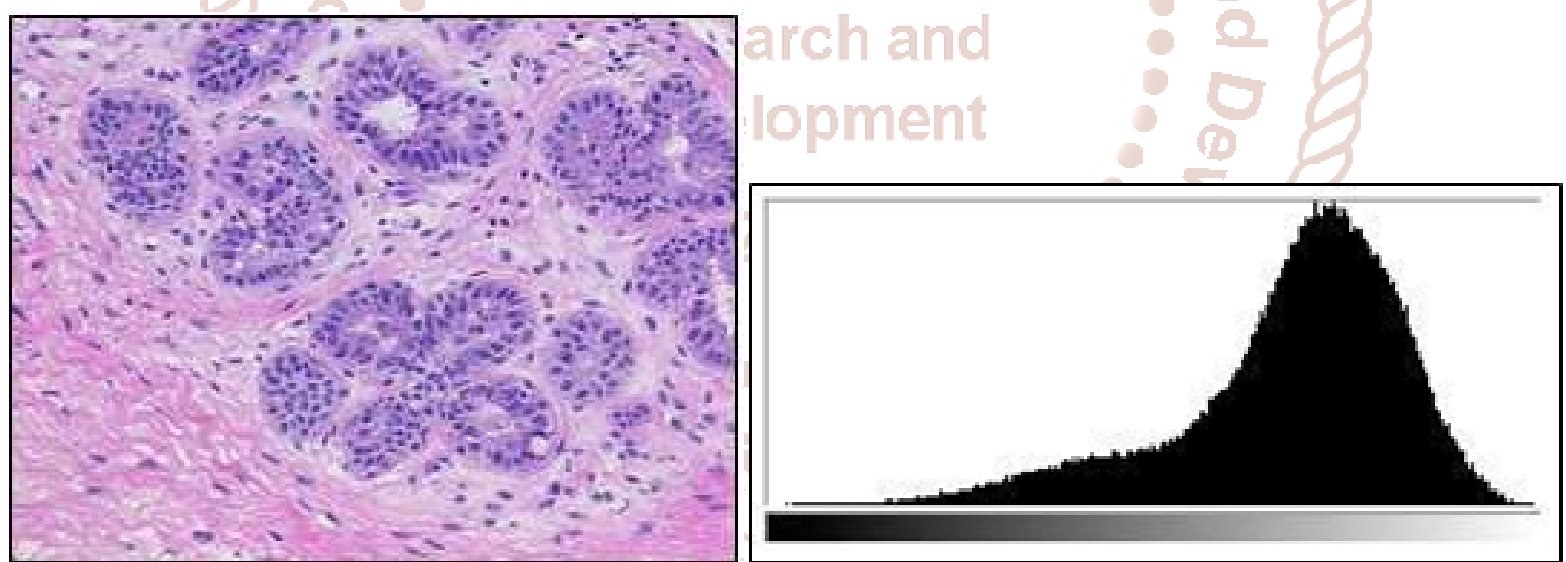

Figure 4 The Original Histopathological Slide showing Normal Cells along with Histogram
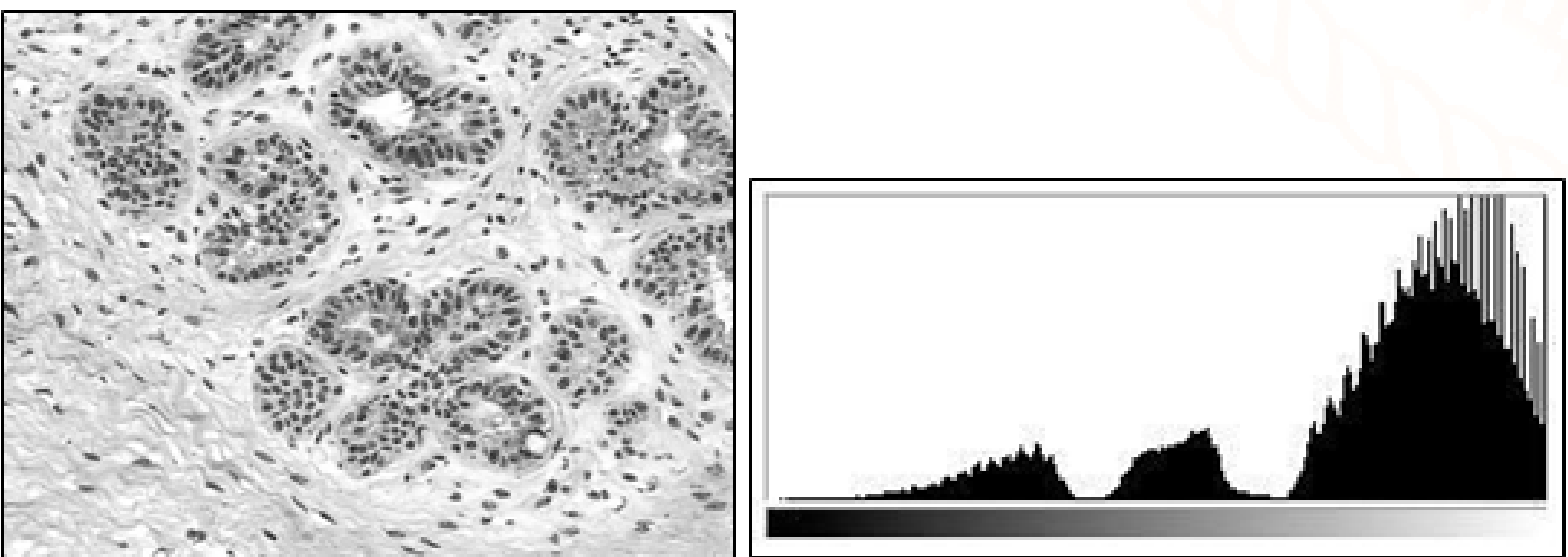

Figure 5 The Grey Shade Histopathological Slide showing Normal Cells along with Histogram 

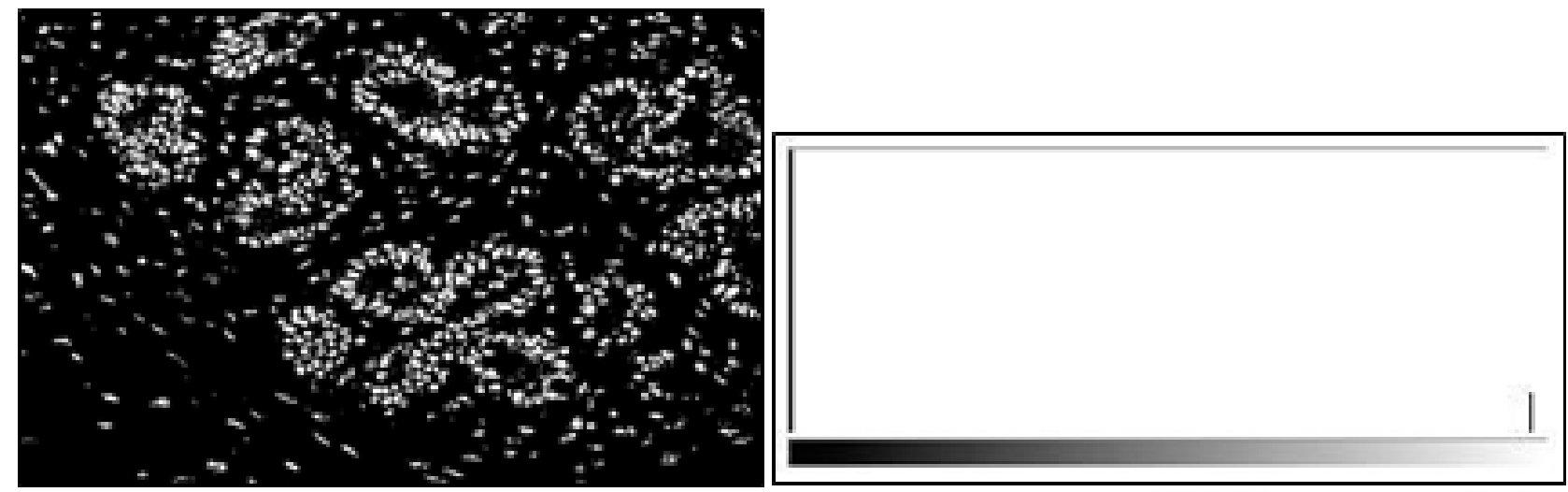

Figure6. Inverse Color Polarized Histopathological Slide showing No abnormalities along with Histogram

Figure 1, 2 and 3 show the Cancerous tissue. In final Figure 3 all the fat, connective tissue and gland tissue are suppressed. It is only showing the parts, which are important to determine the carcinoma. It is depicting the abnormal orientation of malignant cell, shape of the cell and duct. In contrast, Figure 4, 5 and 6, show the normal slide and all the fat, connective tissue, and gland tissues are suppressed in Figure 6. The orientation, shapes etc. in this figure are normal in nature and showing no sign of carcinoma or abnormalities. The image histograms are showing the colour clustering on the basis of algorithms. The primary objective is to suppress less or insignificant parts from the considerable portion of the slide image which is done by the contrast enhancement. For prominence and clustering the similar tissue cells colour polarization is done. The polarization of colour is highly proved by the aforesaid histograms provided.

\section{Conclusions}

In this paper, it is tried to amalgamate screening technology along with histopathological slide image analysis to make it a complete diagnosis tool to assist the experts. The histopathological slide analysis is described in this chapter is a simple and preprocessing step by applying well established methods.

\section{References}

1. Belhomme et al, "Towards a computer aided diagnosis system dedicated to virtual microscopy based on stereo logy sampling and diffusion maps", Diagnostic Pathology, Vol. 6, Issue-S3, pp. 1-4, 2011.

2. Campanini et al, "A novel featureless approach to mass detection in digital mammograms based on Support Vector Machines", Physics in medicine and Biology, Vol. 49, Issue-6, pp. 961-975,2004.
3. Chengetal, "Computer-aided Detection and Classification of Micro-calcifications in Mammograms: A Survey", Pattern Recognition, Vol. 36, Issue-12, pp. 2967-2991, 2003.

4. Etehad Tavakol et al, "Application of K- and Fuzzy c-Means for Color Segmentation of Thermal Infrared Breast Images", Springer Journal of Medical Systems, Vol.34, pp.35-42, 2010.

5. Hartigan et al, "A k-means clustering algorithm", Journal of Applied Statistics, Vol. 28, pp. 100$108,1979$.

6. Fung et al, "Mammographic Determination of Breast Volume by Elliptical Cone Estimation", World Journal of Surgery, Vol. 34, Issue-7, pp.1442-1445, 2010.

7. Jiang et al, "Automated Detection of Breast Mass Spiculation Levels and Evaluation of Scheme Performance", Academic Radiology, Vol. 15, Issue-12, pp. 5341-1544, 2008.

8. Li Liu et al, "Breast density classification using histogram moments of multiple resolution mammograms", Biomedical Engineering and Informatics (BMEI), 3rd International Conference, pp. 146 - 149, 2010.

9. Belhomme et al, "Towards a computer aided diagnosis system dedicated to virtual microscopy based on stereo logy sampling and diffusion maps", Diagnostic Pathology, Vol. 6, Issue-S3, pp. 1-4, 2011.

10. Bahlmann et al, "Automated Detection of Diagnostically Relevant Regions in H\&E Stained Digital Pathology Slides", Proceedings SPIE 8315 Medical Imaging2012, Computer-Aided Diagnosis, 23 Feb, 2012. 